\title{
Short Range Order Modeling in Alloys
}

\author{
Edward Kremer ${ }^{1}$ \\ Independent Researcher
}

\section{Abstract}

The Short and Long Range Orders in alloys can be considered based on a new expression for the combinatorial factor. This expression is more convenient and intuitive than the traditionally used form and can be directly applied to reproduce the results of several good known statisticalthermodynamic models that usually are considered completely independent or even inconsistent.

The short list includes quasichemical theory, associated solution model, surrounded atom model, cluster site approximation.

As result, the formalism and interpretation of these models are significantly clarified, allowing simultaneously to identify and fix several long standing errors that otherwise could be left unnoticed.

Multicomponent generalization of listed models is also critically simplified.

For the systems experiencing a phase transition, the advanced version of theory provides a mechanism allowing to reproduce the correct critical temperature of conversion and at the same time to increase significantly the precision of thermodynamic functions.

\footnotetext{
${ }^{1}$ E-mail: edk137@gmail.com
} 
In the case of the order-disorder transformations, the new theory ensures an integrated description of short and long range orders - a task that has long been considered an important and complex problem.

Keywords: Thermodynamic modeling, Short and Long Range Order Integration, Combinatorial Factor, Order-Disorder Transformations

\section{Introduction}

Short Range Order (SRO) in alloys is in a broad sense a physical-chemical phenomenon, which we associate with deviations in the arrangement of alloy atoms from a chaotic (random) distribution.

There are several types of SRO Ossi (2010), but in this article, we are particularly interested in the chemical SRO Gusev et al. (2001), which manifests itself in any alloy and is caused by distinctions in the physical and chemical properties of components.

There is a clear consensus on the substantial role of SRO in the thermodynamics of liquid and solid alloys Gusev et al. (2001); Sundman et al. (2018); Krivoglaz and Smirnov (1964); Banerjee and Mukhopadhyay (2010). At the same time, the accurate description of SRO presents evident mathematical challenges confirmed by the fact that we do not have a commonly accepted method to take this phenomenon into account.

The most important way to handle this task is the Cluster Variation Method (CVM) Morán-López and Sánchez (2012) that allows taking in the account both short and long range orders and their interrelation.

Unfortunately, the use of this method is limited due to its computational 
complexity, which increases exponentially with the number of components.

Short range parameters Ossi (2010); Gusev et al. (2001); Girifalco (2000) are widely used to describe the elements of SRO accessible from the diffraction experiments, but, unfortunately, provide merely a one-dimensional picture of SRO, that hardly can be considered as sufficient. Details of the three-dimensional arrangement of atoms, which are equally important for understanding the structure of the alloy and which directly affect the thermodynamic and other properties, remain hidden.

While a mathematically satisfactory three-dimensional description of SRO is missing, we have an abundance of attempts to take SRO into the account within the framework of model theories Soustelle (2015); Lupis (1983).

Model theories try to represent SRO by direct modeling the allocation of components within small groups of atoms, and then by calculating the associated with such micro-configurations contributions to the energy and entropy of the alloy. The collected information is used to predict the thermodynamics of the system as a whole.

The described scenario, in which we deduce the thermodynamics of the entire system by just modeling the local atom's arrangement is so typical that it can be used to describe the corresponding theories from some general point of view.

The purpose of this article is to show that indeed, although such theories as Quasichemical Theory Girifalco (2000), Associated Solution Model Soustelle (2015), Surrounded Atom Model Lupis (1983), Cluster Site Approximation Oates and Wenzl (1996) (to name just a few best known) are usually presented in intentionally incompatible forms, they still have a com- 
mon basis, which allows us to treat these models as part of some general approach.

Theory of Inhomogeneous Short Range Order (TISR) Kremer (1988, 2018a) was just developed as an attempt to present the thermodynamics of alloy through the properties and relative amounts of small groups of atoms (cells, as they will be referred to in the following text) conventionally selected in the system, and in this respect is very similar to the listed model theories.

It is distinct however in several aspects, and it is important to recognize the most significant differences when reading this report.

First, it will be provided a new form of treatment for combinatorial problems. It is more intuitive than, for example, the methods traditionally used by the quasichemical theory, and, besides, presents the combinatorial factor in a form that simplifies the multicomponent generalizations.

Second, a new set of variables will be used to express the properties and composition of an alloy in a parametric form. The use of these variables can greatly simplify mathematical formalism.

Third, all versions of formalism will be evaluated by comparison with the results presented for the Ising model in Domb (1974), to be sure that acceptable level of precision can be ensured. This step is vital for eliminating the errors in the combinatorial factor made by some good known model theories, as shown in Kremer (2019) and discussed below.

The presentation of alloy as a conglomerate of cells has many technical advantages.

Each cell makes some contribution to the energy of the system.

The permutations of atoms within the cells and rearrangements of cells 
as a whole are responsible for the configurational entropy of mixing.

At the same time, knowledge of atom allocation inside every cell can be considered as a specific form of SRO presentation in the vicinity of this cell.

When SRO is understood in this sense, it suggests also that SRO is varying from cell to cell.

The term "inhomogeneous" in the name of theory just emphasizes that the model takes into account these local variations of SRO.

The standard short range parameters Ossi (2010); Gusev et al. (2001); Girifalco (2000) use the averaged form of this information and therefore can be used for energy evaluation only.

The ability to calculate the configurational entropy is lost, and usually it means that the crude Bragg-Williams approximation has to be used instead.

TISR shares the described form of SRO presentation with all mentioned model theories and, consequently, it occurs that the developed formalism can be directly applied to reproduce all the results of these theories, if the cells are selected accordingly.

Guided by these principles, in most cases, we can significantly simplify and improve the reconstructed through TISR models.

The advantages of the unification achieved in this way should not be overlooked either.

In the following sections, a detailed explanation of the basic formalism of TISR will be presented and then applied to a few particular cases. Unfortunately, it is impossible to describe within one article all the nuances of the suggested approach.

The bibliography references several preprints that may clarify some of the 
omitted details.

Some issues deserve a separate consideration and will be discussed elsewhere.

\section{Theory of Inhomogeneous Short Range Order}

We start with the simple case where $N$ atoms of an alloy are distributed over the sites of a regular lattice with a coordination number $z$.

Our main goal is to obtain an expression for the free energy of such an alloy. A necessary step in this direction is finding a good approximation for the combinatorial factor of the system.

It is where TISR suggests a new heuristic method that seems to be more intuitive and more productive than, for example, the canonical method used by quasichemical theory Guggenheim (1952) to handle alloy as an ensemble of atom pairs.

The quasichemical theory tries to take into account simultaneously all $z N / 2$ pairs of the nearest neighbors existing in the alloy, plus all permutations of these pairs.

Such an approach is counter-intuitive since the pairs overlapping makes this procedure confusing.

TISR selects atom groups differently, to avoid entirely the overlapping. Namely, rather than consider all possible pairs, one can select a representative subset in which the selected groups have no common elements at all.

Let us follow this scenario, which will help to develop a more intuitive approach.

It is possible from the very beginning to consider cells (atom groups) of 


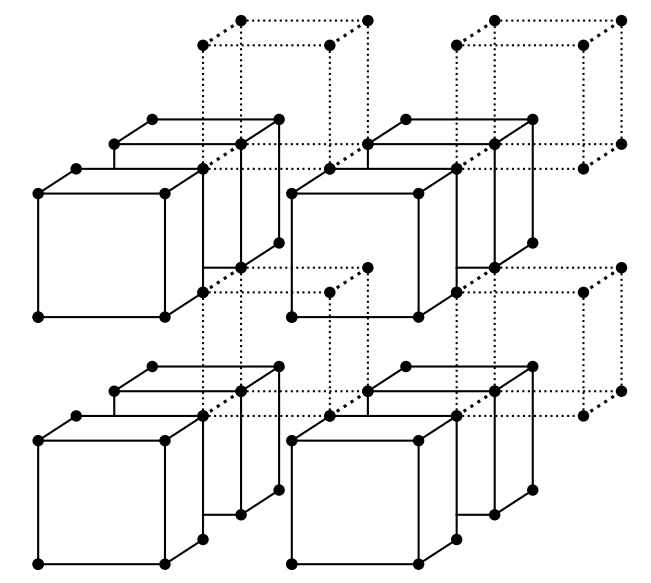

Figure 1: Splitting the grid into cells.

arbitrary size to overcome the good known restrictions of the quasichemical approximation Kremer (2018b).

In Fig. 1 it is presented, as an example, the simple cubic lattice as an ensemble of $2 \times 2 \times 2$ atom groups - cells. Interatomic links between the atoms included in a cell are also included in the cell and are shown in the figure by solid lines. Links between distinct cells are neglected and are shown by dotted lines. Every lattice atom is included in some cell and only in one cell - cells do not intersect.

For the lattice having $N=N_{1}+N_{2}$ sites, we will have $M=N / k$ cells (where $k=8$ in the case under consideration).

The cells can differ in composition, and with the same composition, in the arrangement of the constituent atoms.

It is convenient to describe the values associated with a cell by a pair of 
indexes $i, \alpha$, where the first index implies that the cell has $i$ atoms of type 1 (and, correspondingly, $k-i$ atoms of type 2) and the second index numbers all possible for the cell of specified composition micro-configurations:

$$
\alpha=1,2, \ldots\left(\begin{array}{c}
k \\
i
\end{array}\right)
$$

In particular, let us denote $M_{i \alpha}$ the number of cells of type $i, \alpha$ and $U_{i \alpha}-$ the internal energy of such cell (meaning the energy of $m=12$ links belonging to the cell). Then we can express the total energy $E$ of all links in the system as

$$
E=\beta \sum_{i \alpha} U_{i \alpha} M_{i \alpha}
$$

where $\beta$ is the ratio of the total count of links in the system $z N / 2$ to the count $m M$ of links located inside the described ensemble of cells:

$$
\beta=\frac{z N / 2}{m M}
$$

The introduced symbols can be used to express the composition of alloy:

$$
\begin{aligned}
N_{1} & =\sum_{i \alpha} i M_{i \alpha} \\
N_{2} & =\sum_{i \alpha}(k-i) M_{i \alpha}
\end{aligned}
$$

The expression for the combinatorial factor Hill (1956) is more complicated and will be constructed in several steps.

The starting point is the Bragg-Williams approximation Hill (1956) that neglects all correlations and assumes that the system allows unrestricted atom permutations:

$$
K_{0}=\frac{N !}{N_{1} ! N_{2} !}
$$


We will take this formula as the basic approximation.

The interatomic interactions cause deviations in the atom distribution from the chaotic order, and if we want to take these deviations into the account, we should consider only the macro-configurations that are consistent with this modified distribution.

Mathematically, this means that we are switching from rearranging individual atoms to rearranging entire cells:

$$
K_{1}=\frac{M !}{\prod_{i \alpha}\left(M_{i \alpha}\right) !}
$$

We would like to treat this expression as an alternative combinatorial factor but for this purpose we must be sure that it satisfies two conditions:

- It achieves maximal value when atoms are distributed chaotically;

- It is correctly normalized at this condition, providing the same value as equation (5).

To be sure that the chaotic distribution in the system is achieved, we will consider the high-temperature limit, when the role of energy contribution (1) can be neglected and each lattice site is populated independently of other sites. Then:

$$
M_{i \alpha}^{\infty}=M x_{1}^{i} x_{2}^{k-i}
$$

where the superscript $\infty$ indicates the high-temperature limit and $x_{j}=N_{j} / N$ is the molar fraction of component $j$. We can now use this expression and 
equations (3) - (4) to calculate the value of $K_{1}^{\infty}$ :

$$
\begin{aligned}
\ln K_{1}^{\infty} & =M \ln M-\sum_{i \alpha} M_{i \alpha}^{\infty} \ln M_{i \alpha}^{\infty} \\
& =M \ln M-\sum_{i \alpha} M_{i \alpha}^{\infty}\left(\ln M+i \ln x_{1}+(k-i) \ln x_{2}\right) \\
& =-N_{1} \ln x_{1}-N_{2} \ln x_{2}=\ln K_{0}
\end{aligned}
$$

We just showed that $K_{1}$ is an acceptable combinatorial factor.

We can make one step more to improve this result.

Returning to Fig. 1 we can say that the combinatorial factor $K_{1}$ takes into account the atom correlations associated with the links localized inside cells. The links outside of cells are completely neglected.

We can see also that these neglected links (dotted lines in the picture) form by itself a set of $2 \times 2 \times 2$ cells that is in any respect similar to the original set. Naturally, we want to include correlations associated with this second cell set into the combinatorial factor.

The transition from the Bragg-Williams approximation $K_{0}$ to $K_{1}$ can be represented formally as the application of some multiplicative correction factor to $K_{0}$ :

$$
K_{1}=K_{0} *\left(\frac{K_{1}}{K_{1}^{\infty}}\right)=K_{0} *\left(\frac{K_{1}}{K_{0}}\right)
$$

Since we have two sets of cells we would like to accept the following expression for the combinatorial factor:

$$
K=K_{0} *\left(\frac{K_{1}}{K_{0}}\right)^{\gamma}
$$

where $\gamma=2$ for the case under consideration.

In the general case, the value of $\gamma$ can be estimated similar to the way the $\beta$ value was evaluated: for the case depicted in Fig. 1 one set of cells is 
responsible for $m M$ links belonging to this set; we need $\gamma$ sets to ensure that all $z N / 2$ links are taken into account:

$$
\gamma=(z N / 2) /(m M)
$$

In our case $m=12$, so we receive again that $\gamma=\beta=2$.

For the tetrahedron cell in the face-centered cubic structure $\gamma=\beta=4$.

We have now all the necessary elements to construct the expression for the free energy:

$$
\begin{aligned}
F & =\beta \sum_{i \alpha} U_{i \alpha} M_{i \alpha}-\theta(1-\gamma) \ln K_{0}-\theta \gamma \ln K_{1} \\
& +\lambda_{1}\left(N_{1}-\sum_{i \alpha} i M_{i \alpha}\right)+\lambda_{2}\left(N_{2}-\sum_{i \alpha}(k-i) M_{i \alpha}\right)
\end{aligned}
$$

where $\theta=k_{B} T$ is a temperature factor - the product of absolute temperature $T$ and the Boltzmann constant $k_{B}$ Hill (1956); $\lambda_{1}$ and $\lambda_{2}$ are the Lagrange multipliers Hill (1956).

Taking into account that

$$
\ln K_{1}=M \ln M-\sum_{i \alpha} M_{i \alpha} \ln M_{i \alpha}
$$

we can get now the basic equations of the model:

$$
\frac{\partial F}{\partial M_{i \alpha}}=\beta U_{i \alpha}+\theta \gamma \ln p_{i \alpha}-i \lambda_{1}-(k-i) \lambda_{2}=0
$$

where $p_{i \alpha}=M_{i \alpha} / M$.

The solution to these equations can be written immediately as

$$
p_{i \alpha}=\exp \left(-\frac{\beta U_{i \alpha}}{\theta \gamma}\right) b_{1}^{i} b_{2}^{k-i}
$$


where $b_{j}$ are defined by

$$
\lambda_{j}=\theta \gamma \ln b_{j}, \quad j=1,2
$$

Taking into account the explicit dependency of $K_{0}$ on $N_{j}(5)$ and the expression for the free energy (10), we can calculate now the chemical potentials $\mu_{j}$ of components:

$$
\mu_{j}=\frac{\partial F}{\partial N_{j}}=\theta(1-\gamma) \ln x_{j}+\lambda_{j}=\theta \ln x_{j}+\theta \gamma \ln \frac{b_{j}}{x_{j}}
$$

We must introduce some additional notations that will be needed to represent all physically important objects in a compact form:

$$
p_{i}=\sum_{\alpha} p_{i \alpha}=W_{i} b_{1}^{i} b_{2}^{k-i}
$$

where

$$
W_{i}=\sum_{\alpha} \exp \left(-\frac{\beta U_{i \alpha}}{\theta \gamma}\right)
$$

$W_{i}$ can be treated as the statistical sum Hill (1956) of a cell containing $i$ atoms of type 1 (and $k-i$ atoms of type 2 ).

Even if the factor $\beta / \gamma$ in this expression is exactly equal to one, we keeping it in place to have the possibility to make a more sophisticated choice of $\gamma$ in the following sections.

The equations (3) and (4) can be rewritten now in a more compact form:

$$
\begin{gathered}
\sum_{i} p_{i}=b_{2}^{k} \sum_{i} W_{i} c^{i}=1 \\
\sum_{i} i p_{i}=b_{2}^{k} \sum_{i} i W_{i} c^{i}=k x_{1}
\end{gathered}
$$


where the new notation $c$

$$
c:=b_{1} / b_{2}
$$

was introduced to be used as an independent variable for the parametric representation of both the composition and the thermodynamic activities of components.

First, equation (17) defines $b_{2}$ as a function of $c$. Then the equation (19) defines $b_{1}$ as a function of $c$. This information can be directly substituted into expressions for $x_{j}$ and $\mu_{j}$ completing the calculation.

The necessary basis for the described calculation is the knowledge of $W_{i}$, which are expected to be considered as the model parameters in the applications of theory.

All formulas in this section were derived in the assumption that we consider a regular lattice with a specific coordination number, however all the details of the atom arrangements and interaction occurred hidden inside the parameters $W_{i}$. It means not only a very significant economy in the number of adjustable parameters but also the opportunity to extend the theory outside the original assumptions. The atoms distribution inside cells may be irregular but we hope that this irregularity can be encapsulated into parameters describing cells.

This consideration helps to justify the commonly used applications of quasi-lattice approximation to liquid mixtures Soustelle (2015).

Numerous examples of using the model to describe the thermodynamic functions of binary systems can be found in $\operatorname{Kremer}(2018 \mathrm{a}|\mathrm{b}| \mathrm{c})$. 


\section{Quasichemical Theory}

The Quasichemical Theory (QT) corresponds to the selection of dimers $(k=2)$ as cells. The equations of the previous section can be applied to this case directly without any modifications. All the equations $100-(19)$ remain unchanged but with different value for $\beta=\gamma=z)$.

It can be directly shown Kremer (2018b) that the TISR expressions for energy (1) and combinatorial factor (8) coincide with the standard expressions known from QT Guggenheim (1952), with perhaps a slight difference in notations.

The resulting expressions for thermodynamic functions are represented by TISR in parametric form and therefore look distinct from the expressions found in Hill (1956) or Guggenheim (1952).

We have to get rid of parametric representation and convert the equations to the explicit form to confirm that the results of both theories are exactly the same.

To do this, it is necessary to take into account that in the case of $k=2$ we have only one not trivial (distinct from 1) value for $W_{i}$ :

$$
\begin{aligned}
& U_{0 \alpha}=U_{2 \alpha}=0 ; \quad U_{1 \alpha}=U_{\Delta} \\
& W_{0}=W_{2}=1 ; \quad W_{1}=2 \exp \left(-\frac{U_{\Delta}}{\theta}\right) \rightleftharpoons 2 \eta
\end{aligned}
$$

since we care here only about thermodynamic functions of mixing. It was taken into account here that $\gamma=\beta$ and therefore both can be excluded from the expression for $W_{1}$.

Using these values and the equations (17) and (18) we can receive the equation defining the parameter $c$ : 


$$
\frac{\eta c+c^{2}}{1+\eta c}=\frac{x_{1}}{x_{2}}
$$

Getting the value of $c$ from this quadratic equation we can substitute it into the expression for the chemical potential (14), immediately reproducing Kremer (2018b) the standard quasichemical expressions Hill (1956); Guggenheim (1952):

$$
\mu_{i}=\theta \ln x_{j}+\theta \gamma \ln \frac{b_{j}}{x_{j}}=\theta \ln x_{i}+\frac{\theta z}{2} \ln \frac{r-1+2 x_{i}}{x_{i}(r+1)}
$$

where

$$
r^{2}=\left(x_{1}-x_{2}\right)^{2}+\frac{4 x_{1} x_{2}}{\eta^{2}}
$$

The results of this section show that the simplest version of TISR ensures the precision of consideration not worse than by quasichemical theory. We can expect that the usage of cells with $k>2$ will only improve the quality of the approximation.

\section{Improved Combinatorial Factor}

The introduced in section 2 parameters $\gamma$ and $\beta$ turned out to have the same value, as defined by (2) and (9).

While the choice of $\beta$ looks quite good founded, the selection of $\gamma$ may raise some questions.

In fact, the final value of $\gamma$ in $(9)$ is based solely on the assumption that the distribution of links within one ensemble of cells is completely independent of the existence of other similar ensembles. 
In classical literature devoted to QT this type of assumption was named "non-interference hypothesis" Guggenheim (1952).

This hypothesis looks quite plausible at high temperatures but hardly could be justified in the critical region where the correlation length aims to infinity.

It can be expected that in the proximity to the critical point of miscibility the knowledge of correlations associated with one cell ensemble provides at least partial information about correlations associated with other ensembles.

Effectively, it may mean that near the critical point the effective value of $\gamma$ could be less than it is suggested by the "non-interference hypothesis" and defined by expression (9).

Unfortunately, there are no simple approaches to assess how the effect under discussion can affect the value of $\gamma$.

It turns out however Kremer (2019) that it is quite possible to impose on $\gamma$ an additional requirement, which will help to ensure the reproduction of the correct critical temperature of immiscibility and at the same time significantly improve the calculated values of the most important thermodynamic quantities.

This $\gamma$ adjustment can be easily done within the framework of the Ising model - i.e. the environment that provides a comprehensive list of data related to the critical region Domb (1974) and therefore a good reference base for comparison.

To handle this task we need a few simple formulas related to immiscibility phenomenon.

Under the standard assumptions of the Ising model Hill (1956), Domb 
(1974) the curve representing the dependency of free energy on the composition is symmetrical with respect to the concentration $x=1 / 2$.

It means also that the immiscibility curve (binodal Girifalco (2000)) can be described as not trivial $(x \neq 1 / 2)$ solution of the equation

$$
\frac{\partial f}{\partial x_{1}} \equiv \mu_{1}\left(x_{1}\right)-\mu_{2}\left(x_{1}\right)=0
$$

Using equation (14) for the chemical potential and definition (19) we can interpret (23) as an equation on $c$ :

$$
\frac{\partial f}{\partial x_{1}}=\theta(1-\gamma) \ln \frac{x_{1}}{x_{2}}+\theta \gamma \ln c=0
$$

or

$$
\frac{x_{1}(c)}{x_{2}(c)}=c^{\frac{\gamma}{\gamma-1}}
$$

Assuming that $\gamma$ is known, this transcendent equation defines $c$ and the corresponding points on the binodal as functions of temperature $\theta$.

Critical point corresponds to $x_{1}=0.5$ when $c=1$. At this condition the equations (25) turns into identity. Resolving this uncertainty we coming to the final expression for the critical point:

$$
\frac{\gamma}{\gamma-1}=\lim _{c \rightarrow 1} \frac{x_{1}(c)-x_{2}(c)}{(c-1) x_{2}(c)}
$$

Using data from (Domb (1974) $)$ and the expression 18) for $x_{1}$, this transcendent equation can be solved for multiple two- and three-dimensional Ising models and approximations. The results are collected in table 1 . 
Table 1: Critical Values (per atom) calculated using Bragg-Williams (BW), Surrounded Atom Model (SAM), Quasichemical (QT), and Improved Quasichemical Theory (IQT) Kremer (2019) along with the best theoretical estimations Domb 1974)

\begin{tabular}{|c|c|c|c|c|c|c|c|}
\hline LATTICE & & BW & SAM & QT & IQT & IQT2 & Domb \\
\hline \multirow{5}{*}{$2 d$ square } & $k$ & 1 & 5 & 2 & 2 & 4 & \\
\hline & $\gamma$ & 1 & 5 & 4 & 2.784 & 1.608 & \\
\hline & $U_{\Delta} / \theta_{c}$ & 0.5 & 0.575 & 0.693 & 0.882 & 0.882 & 0.882 \\
\hline & $E / U_{\Delta}$ & 1.000 & 0.857 & 0.667 & 0.439 & 0.412 & 0.292 \\
\hline & $S / k_{B}$ & 0.693 & 0.652 & 0.580 & 0.461 & 0.430 & 0.306 \\
\hline \multirow{5}{*}{$2 d$ triangular } & $k$ & 1 & 7 & 2 & 2 & 3 & \\
\hline & $\gamma$ & 1 & 7 & 6 & 3.002 & 1.956 & \\
\hline & $U_{\Delta} / \theta_{c}$ & 0.333 & 0.365 & 0.405 & 0.549 & 0.549 & 0.549 \\
\hline & $E / U_{\Delta}$ & 1.5 & 1.364 & 1.2 & 0.751 & 0.715 & 0.501 \\
\hline & $S / k_{B}$ & 0.693 & 0.668 & 0.633 & 0.497 & 0.471 & 0.330 \\
\hline \multirow{5}{*}{ cubical } & $k$ & 1 & 7 & 2 & 2 & 8 & \\
\hline & $\gamma$ & 1 & 7 & 6 & 4.44 & 1.673 & \\
\hline & $U_{\Delta} / \theta_{c}$ & 0.333 & 0.365 & 0.405 & 0.443 & 0.443 & 0.443 \\
\hline & $E / U_{\Delta}$ & 1.5 & 1.364 & 1.2 & 1.064 & 1.051 & 0.966 \\
\hline & $S / k_{B}$ & 0.693 & 0.668 & 0.633 & 0.598 & 0.588 & 0.541 \\
\hline \multirow{5}{*}{$\mathrm{BCC}$} & $k$ & 1 & 9 & 2 & 2 & 4 & \\
\hline & $\gamma$ & 1 & 9 & 8 & 5.234 & 2.729 & \\
\hline & $U_{\Delta} / \theta_{c}$ & 0.250 & 0.267 & 0.288 & 0.315 & 0.315 & 0.315 \\
\hline & $E / U_{\Delta}$ & 2.000 & 1.867 & 1.714 & 1.528 & 1.524 & 1.452 \\
\hline & $S / k_{B}$ & 0.693 & 0.675 & 0.652 & 0.619 & 0.617 & 0.585 \\
\hline \multirow{5}{*}{ FCC } & $k$ & 1 & 13 & 2 & 2 & 4 & \\
\hline & $\gamma$ & 1 & $1 \beta_{8}$ & 12 & 5.845 & 2.566 & \\
\hline & $U_{\Delta} / \theta_{c}$ & 0.166 & 0.174 & 0.182 & 0.204 & 0.204 & 0.204 \\
\hline & $E / U_{\Delta}$ & 3.000 & 2.870 & 2.727 & 2.381 & 2.362 & 2.270 \\
\hline & $S / k_{B}$ & 0.693 & 0.682 & 0.668 & 0.63 & 0.623 & 0.597 \\
\hline
\end{tabular}




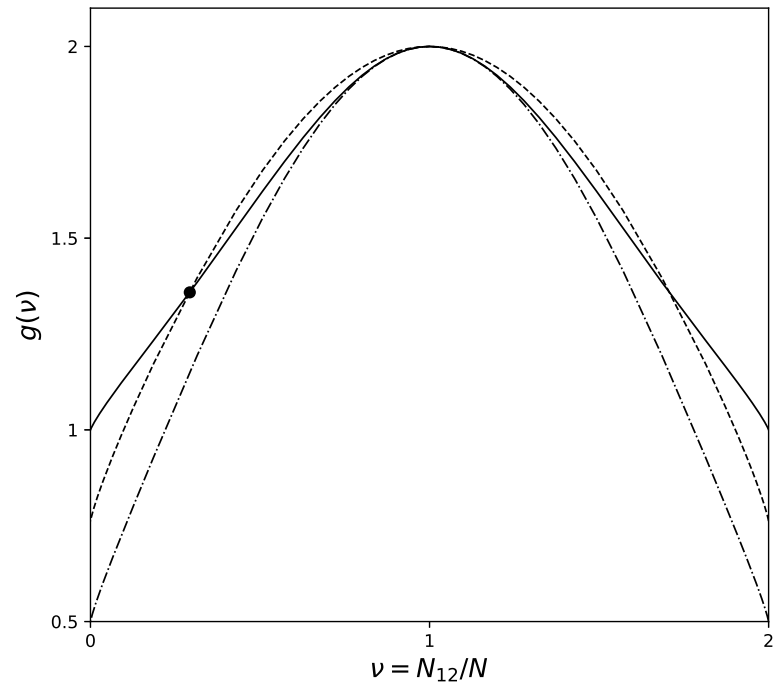

Figure 2: Versions of Combinatorial Factor $g(\nu)$ for Square $2 d$ lattice:

Quasichemical approximation - dash-dot line

Improved Quasichemical approximation - dashed line

Onsager solution Onsager (1944) - solid line

Location of the critical point by Onsager (1944) - large dot 
The data in the table show that the "Improved Quasichemical Theory" (IQT) really provides significant improvements compared to the standard Quasichemical Theory (QT).

The possibility to completely eliminate the error in determining the location of the critical point in the phase diagram should be considered extremely important if one remembers that the usual quasichemical $10 \%$ shift in the critical temperature value can mean a defect up to $100^{\circ}$ (or even more) for typical metal alloys.

Data in the table show also that the adjustment of the critical temperatures helps at the same time to reduce significantly the errors in estimating the critical values of energy and entropy of the alloy.

This fact can be confirmed and clarified by Fig. 2, which shows two different versions of the $\mathrm{CF}$ at $x=1 / 2$, compared to the exact $\mathrm{CF}$ derived by Prigogine et al. (1952) using the Onsager solution Onsager (1944) for the square lattice.

High temperature corresponds to chaotic atom distribution (equivalent to $\nu=1$ for the square lattice) and we can see from the picture that in this region the ordinary QT approximation is extremely close to the Onsager's solution.

When the temperature decreases and we approach the critical point of mixing, then the difference between QT and the exact curve sharply increases.

On the contrary, partially losing the quality of approximation in the hightemperature limit, IQT provides a more uniform (though hardly perfect) representation of the exact curve over the entire range from high temperatures to the critical point. 


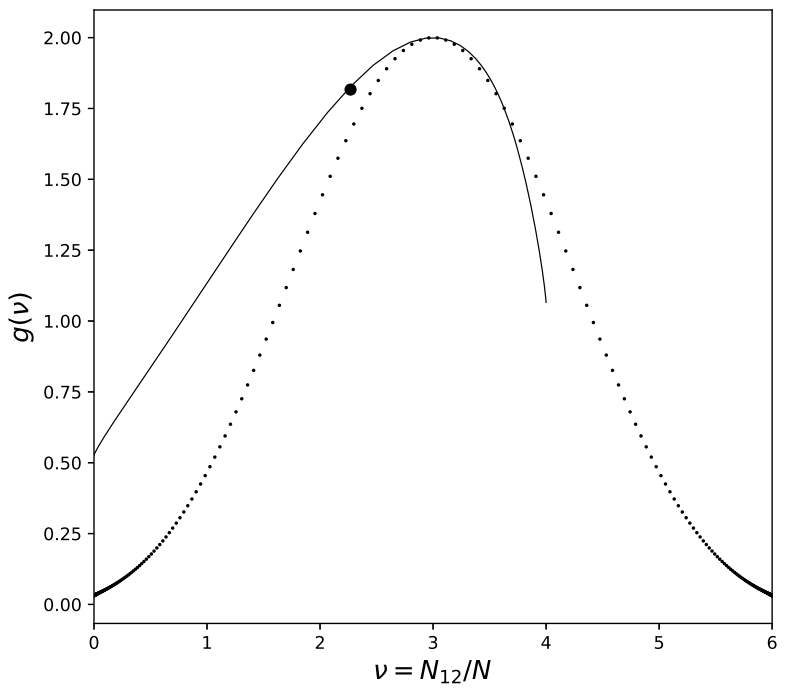

Figure 3: Versions of Combinatorial Factor $g(\nu)$ for FCC Lattice:

Quasichemical approximation - dotted line

Improved Quasichemical approximation with $k=4$ - solid line

Location of the critical point by Domb (1974) - large dot

Exaggerating, we can say that QT is very good around one point, while IQT is reasonably good inside the whole physically meaning region. This is the real reason why all the related values improve when we switch from QT to IQT.

In the above part of this section, we considered the systems with positive deviation from Raoult's Law Lukas (2007) only. The atoms in these systems prefer to be surrounded by atoms of the same type, especially when the temperature drops and approaches the critical point.

Atoms in systems with negative deviations from Raoult's law tend to be surrounded by atoms of the opposite kind, but this tendency can be severely limited in some cases. 
Let us consider as an example the Face Centered Cubic lattice (FCC). No matter how you divide the lattice into two sublattices, each atom will have neighbors from both sublattices. As result, some part of the links will be of $a-a$ or $b-b$ type and the proportion of $a-b$ pairs can never exceed $2 / 3$, as seen from Fig. 3 .

Such condition is commonly known as "geometrical frustration" Sadoc (1999).

Frustration-free lattices, such as Cubical or Base Centered Cubical, have an additional symmetry Hill (1956), which effectively leads to a symmetric form of CF dependence on the number of pairs $a-b$, as in Fig. 2 .

Frustrated lattices are critically different in this respect but the asymmetrical behavior of CF cannot be recognized on the level of pair-like approximation.

It is one of the multiple limitations of QT, explained in detail in Kremer (2018b).

We need at least four atoms to be included in the cell to present properly the three-dimensional structure of alloy and be sure that the frustration phenomenon is correctly described.

Returning to Fig. 3 we can see that the left branch of CF curve goes above the quasichemical graph. The value of $\gamma$ corresponding to the left branch is less than the quasichemical value, in agreement with the heuristic arguments at the beginning of the section.

The ordering phase transition in the FCC lattice (right branch) is of the first order Krivoglaz and Smirnov (1964) and therefore these arguments cannot be applied. Indeed, it will be shown in section 7.1 that ordering in the 
$\mathrm{Au}-\mathrm{Cu}$ system has to be described by $\gamma$ that is larger than the quasichemical estimation (9).

\section{Surrounded Atom Model}

The Surrounded Atom Model (SAM) was introduced in Mathieu et al. (1965); Hicter et al. (1967) as a direct generalization of quasichemical theory.

The motivation for such generalization was the well-known deficiency of QT Guggenheim (1952), Kremer (2018b) which is not capable to describe systems with any asymmetry on the curve representing the dependence of thermodynamic values on the composition.

The generalization was achieved by replacing the dimers, used as the basis of QT, by groups of $z+1$ atoms, where every group consists of one "central" atom and all $z$ its nearest neighbors. This approach opens the possibility to introduce the direct dependence of atom interactions on the local composition and explain in this way the asymmetry, if any.

Though SAM cannot be considered as popular as QT, it is still in use Lehmann (2008); Lehmann and Zhang (2010); Ha and Khanh (2011); Kotova et al. (2015) because of its intuitive appeal.

The formalism of theory was constructed to mimic the arguments used by Guggenheim (1952) to derive the classical QT equations, but a sad mistake in calculating the combinatorial factor - the basic element of theory significantly impaired the quality of the resulting model.

To describe the "surrounded atoms" in the framework of TISR Kremer (2020a), we selecting as a cell the same configuration as SAM does: atom surrounded by $z$ nearest neighbors. Just as is commonly accepted by SAM, 
only $z$ links between the central atom and the shell are included in the cell; links between the surrounding atoms are excluded.

A central atom in the cell occurs in a special position and we must take extra care Kremer (2020b) to be sure that central and peripheral atoms have the same average composition.

To handle this task, we can present a cell as a combination of two subcells, where the central atom constitutes one sub-cell, while $z$ surrounding atoms are included in other sub-cell.

It is convenient for the following calculations to define the sub-cell that contains $z$ peripheral atoms as the first sublattice $\left(k_{1}=z\right)$. The central atom is then the only element of the second sub-cell $\left(k_{2}=1\right)$.

Thus, the total size of the cell is

$$
k=k_{1}+k_{2}=z+1
$$

To completely describe a cell we must specify the content of every subcell and indicate how atoms are distributed. To do this we will need three indexes, and in the remainder of this section $M_{i j \alpha}$ will mean the number of cells having every:

i atoms of type $a$ in sub-cell 1 ;

$\mathrm{j}$ atoms of type $a$ in sub-cell 2;

$\alpha$ will number all possible configurations of a cell having $i$ atoms of type $a$ in sub-cell 1 and $j$ such atoms in sub-cell 2:

$$
\alpha=1,2, \ldots\left(\begin{array}{c}
k_{1} \\
i
\end{array}\right)\left(\begin{array}{c}
k_{2} \\
j
\end{array}\right)
$$

The combinatorial factor will be still expressed by equation (8) where $K_{1}$ 
is defined this time as

$$
\ln K_{1}=\ln \frac{M !}{\prod_{i j \alpha}\left(M_{i j \alpha}\right) !}=-M \sum_{i j \alpha} p_{i j \alpha} \ln p_{i j \alpha}
$$

Taking into account the existence of sub-cells, we can write down the free energy of the system in the following form:

$$
\begin{aligned}
& F\left(N_{1}, N_{2}, \lambda_{1}, \lambda_{2}, \lambda, p_{i j \alpha}, \theta\right) \\
& =\beta M \sum_{i \alpha} U_{i j \alpha} p_{i j \alpha}-\theta(1-\gamma) \ln K_{0}-\theta \gamma \ln K_{1} \\
& +\lambda_{1} N\left(x_{1}-\sum_{i j \alpha} \frac{i}{k_{1}} p_{i j \alpha}\right)+\lambda_{2} N\left(x_{1}-\sum_{i j \alpha} \frac{j}{k_{2}} p_{i j \alpha}\right) \\
& +\lambda N\left(1-\sum_{i j \alpha} p_{i j \alpha}\right)
\end{aligned}
$$

where we have additional (compared to (10) ) restriction included and

$$
\begin{aligned}
M & =\frac{N}{k}=\frac{N}{z+1} \\
\beta & =\frac{N z}{2 M z}=\frac{z+1}{2}
\end{aligned}
$$

The structural parameter $\gamma$, when taken in the quasichemical approximation Kremer (2018b), is equal to $\beta$

$$
\gamma=\beta=\frac{z+1}{2}
$$

The minimization of the free energy 30 results in

$$
p_{i j \alpha}=\exp \left(-\frac{\beta U_{i j \alpha}}{\theta \gamma}\right) b_{1}^{i} b_{2}^{j} b
$$


where $b$ and $b_{j}$ are defined as

$$
\begin{gathered}
b_{j}=\exp \left(\frac{\lambda_{j}}{\theta \gamma} \frac{k}{k_{j}}\right) \\
b=\exp \left(\frac{\lambda k}{\theta \gamma}\right)
\end{gathered}
$$

As immediately follows from the expression f30 for the free energy,

$$
\begin{aligned}
& \mu_{1}=\frac{\partial F}{\partial N_{1}}=\theta(1-\gamma) \ln x_{1}+\lambda+\lambda_{1}+\lambda_{2} \\
& \mu_{2}=\frac{\partial F}{\partial N_{2}}=\theta(1-\gamma) \ln x_{2}+\lambda
\end{aligned}
$$

So far, we have not imposed any restrictions on the dependence of cell' energy on the composition, that is so important in SAM. But since our primary interest is the behavior of combinatorial factor, from this moment on we will assume that the energy of cells follows strongly the simple rules of the Ising model Girifalco (2000):

$$
U_{i 0 \alpha}=U_{\Delta} i ; \quad U_{i 1 \alpha}=U_{\Delta}(z-i)
$$

where $U_{\Delta}$ is the energy of one link $a-b$.

This assumption cannot influence the combinatorial factor, which depends on the geometrical structure of the lattice only, but significantly simplifies and clarifies our work by reducing both models to the ordinary quasichemical theory (some simplification that is certainly impossible in the general case when the energy of the "surrounded atom" is a nonlinear functional of the composition).

As the result of the assumption (36), and as it follows from (33), we can see immediately that the probabilities $p_{i j \alpha}$ are independent of $\alpha$, so we can 
introduce new variables $p_{i j}$ :

$$
p_{i j}=\sum_{\alpha} p_{i j \alpha}=C_{z}^{i} p_{i j 1}
$$

where, in agreement with the usual SAM notation Mathieu et al. (1965); Hicter et al. (1967):

$$
C_{z}^{i} \equiv\left(\begin{array}{l}
z \\
i
\end{array}\right)
$$

In general, the notations used by SAM and TISR are significantly different and have to be brought to a common basis for comparison.

In particular, the ensemble of "surrounded atoms" consists of $N$ elements, while the TISR' ensemble includes only $N /(z+1)$ elements.

This difference has to be taken in the account when comparing the intermediate values.

The variables describing cells are defined differently inside SAM Kapoor (1978), where $n_{A i}$ denotes the number of atoms $a$ surrounded by $i$ atoms $b$, and $n_{B i}$ denotes the number of atoms $b$ surrounded by $i$ atoms $a$ :

$$
\begin{aligned}
& n_{A i}=N p_{(z-i) 1}=N C_{z}^{i} p_{(z-i) 11} \\
& n_{B i}=N p_{i 0}=N C_{z}^{i} p_{i 01}
\end{aligned}
$$

The critical point, where the difference between TISR and SAM becomes evident, is the expression for the combinatorial factor, namely, the part of the expression for the free energy (30) that includes $K_{1}$ :

$$
\begin{aligned}
\gamma \ln K_{1} & =-\gamma M \sum_{i j \alpha} p_{i j \alpha} \ln p_{i j \alpha} \\
& =-\frac{z+1}{2} \frac{N}{z+1} \sum_{i j} C_{z}^{i} p_{i j 1} \ln p_{i j 1} \\
& =\frac{1}{2}\left(\ln N !-\ln \prod_{i}\left(n_{A i} / C_{z}^{i !} !\right)^{C_{z}^{i}}-\ln \prod_{i}\left(n_{B i} / C_{z}^{i} !\right)^{C_{z}^{i}}\right)
\end{aligned}
$$


This expression contains an extra multiplier 1/2, compared to Kapoor (1978).

The same problem can be indicated in the expression for the internal energy of the system:

$$
\begin{aligned}
E & =\beta M \sum_{i \alpha} U_{i j \alpha} p_{i j \alpha} \\
& =\frac{z+1}{2} \frac{N}{z+1} \sum_{i} C_{z}^{i}\left(U_{i 01} p_{i 01}+U_{i 11} p_{i 11}\right) \\
& =\frac{1}{2} \sum_{i}\left(U_{i 01} n_{B i}+U_{(z-i) 11} n_{A i}\right)
\end{aligned}
$$

Again, we have here extra factor $1 / 2$ that is missing in SAM Kapoor (1978).

It means that the equations of SAM can be formally received from the equations of TISR if we replace the common value of $\gamma=\beta$ by the value that is two times larger.

The source of this difference is easy to understand.

Parameters $\beta$ and $\gamma$ are defined above by conditions (2) and (9), which are actually identical, so we will consider as an example the second equation only:

$$
\gamma(M m)=\frac{z N}{2}
$$

We can say that $\gamma$ is selected to ensure that every link in the system is counted in the combinatorial factor precisely one time.

Combinatorial factor in SAM is constructed Kapoor (1978); Bichara et al. (1985) by treating the "surrounded atoms" as independent entities, rather than pairs.

It looks natural but it is the source of the problem. 
Let us consider an arbitrary pair of neighboring atoms $m-m^{\prime}$. We can identify the "surrounded atom" where $m$ is the central atom and $m^{\prime}$ is located in its shell.

We can consider also the "surrounded atom" where $m^{\prime}$ is the central atom and $m$ is located in its shell.

But it means that when calculating the combinatorial factor, the pair $m-m^{\prime}$ is taken into account twice, while in TISR, as we have seen, every pair is taken into account only once.

As result, the precision of SAM is only intermediate between the precision of Bragg-Williams approximation and precision of quasichemical approximation, as can be seen from table 1 .

At the same time, the described version of TISR ensures a quasichemical level of precision. And it can be significantly improved by refining the combinatorial factor of the system as shown in section 4 .

\section{Associated Solution Model}

Using the equation (15) that defines the probability to find a cell of given composition, we can construct an expression that contains no parameters $b_{i}$ at all and therefore is a function of the temperature only:

$$
\frac{p_{i}^{k}}{p_{0}^{k-i} p_{k}^{i}}=\frac{W_{i}^{k}}{W_{0}^{k-i} W_{k}^{i}}
$$

It looks precisely as the mass action equation for the ideal Association Solution Model (ASM), where cells are treated as "associates". In this sense we can say that TISR is always formally equivalent to some effective ideal ASM. 
At the same time, even if the just derived equilibrium equation 42 looks formally identical to the ordinary equation used by ideal ASM, the two theories occur to be quite different.

First, we can see that the expression for the energy (1) of alloy can be divided in two parts:

$$
E=\sum_{i \alpha} U_{i \alpha} M_{i \alpha}+(\beta-1) \sum_{i \alpha} U_{i \alpha} M_{i \alpha}
$$

where the first term can be treated as formation energy of cells that are considered as "associates".

The second term has to be considered therefore as interaction energy of "associates".

The difference between the two theories will become even clearer if we take into account the specifics of the choice of the combinatorial factor.

The standard for ASM selection of combinatorial factor is based on the Bragg-Williams approximation Hill (1956) being applied to associates, and can be formally derived from the TISR expression (8) at $\gamma=1$.

If we return to the equation (26) that describes the critical point of immiscibility we can see that $\gamma=1$ is the point of singularity at which this equation has no solution.

This remark confirms the good know fact that the ideal ASM is not capable to describe the systems with positive deviations from Raoult's Law Pelton and Kang (2007).

At the same time, it can be shown Kremer (2018c) that the efficient TISR-based ASM corresponds to $\gamma>1$ and is completely free of this and many other problems that are so typical for standard ASM Sundman et al. (2018); Soustelle (2015); Lukas (2007); Pelton and Kang 2007). 
The discussed above possibility to rewrite the equations of TISR in the form similar to the equations of ideal ASM opens the possibility to immediately use TISR for phase diagram calculations since most of the existing phase diagram calculation engines implement ASM.

This approach suggests some transformations associated with equation (42) ensuring a significant reduction in the number of variables to be estimated. This important issue will be considered in detail elsewhere.

Directly related to all mentioned issues is the physical interpretation of ASM equations, which is important, non-trivial, and also will be discussed in detail elsewhere.

\section{Order-Disorder Transformations}

In this section, we will consider crystalline solids that exhibit orderdisorder transformations at low temperatures Lukas (2007), when atoms can be redistributed over several interlaced sublattices.

As could be expected, mathematical description requires some specific in this case. As a working example, we will consider the superstructure $\mathrm{Cu}_{3} \mathrm{Au}$ which appears in the $\mathrm{Cu}-\mathrm{Au}$ system below a certain critical temperature and has a Face Centered Cubical (FCC) lattice Girifalco (2000).

It is good known Girifalco (2000) that the $\mathrm{Cu}_{3} \mathrm{Au}$ superstructure can be presented as the association of two interlaced sublattices, one of which is preferable for $\mathrm{Cu}$ and has three times more nodes than the second sublattice that is preferable for $\mathrm{Au}$.

It is convenient for our consideration to distinguish these sublattices by an index (which, we assume, varies from one to two). 
Natural in our case selection of tetrahedron as the basic cell is resulting in identifying two sub-cells inside every cell.

One of the sub-cells has three nodes and is preferable for $\mathrm{Cu}$, while the second sub-cell has one node and is preferable for $\mathrm{Au}$.

We will assign an index to every sub-cell to be identical with the index of the corresponding sublattice.

The configuration of the system can be described as follows.

The lattice having $N=N_{1}+N_{2}$ knots is divided into $M=N / k$ cells containing every $k=4$ knots.

We need two variables $\left(k_{1}, k_{2}\right)=(3,1)$ to specify the count of knots in each sub-cell:

$$
k=k_{1}+k_{2}
$$

It means that the total number of knots in the sublattice $m$ is

$$
N^{(m)}=k_{m} M=k_{m} N / k, \quad(m=1,2)
$$

To describe a cell we must specify the content of every sub-cell. We will use for this purpose two indexes $i, j$.

Accordingly, $M_{i j \alpha}$ is the number of cells having every:

$i$ atoms of type $a$ in sub-cell 1;

$j$ atoms of type $a$ in sub-cell 2;

index $\alpha$ numbers all possible configurations for the cell of specified composition:

$$
\alpha=1, \ldots\left(\begin{array}{c}
k_{1} \\
i
\end{array}\right)\left(\begin{array}{c}
k_{2} \\
j
\end{array}\right)
$$


In the following part of this section, the superscript indicates the sublattice, while the subscript - the component's number.

In particular, the total number $N_{1}^{(m)}$ of atoms a belonging to the sublattice $m$ is:

$$
\begin{aligned}
& N_{1}^{(1)}=\sum_{i j \alpha} i M_{i j \alpha} \\
& N_{1}^{(2)}=\sum_{i j \alpha} j M_{i j \alpha}
\end{aligned}
$$

with the total number of atoms $a$ in the system:

$$
N_{1}=\sum_{m=1}^{2} N_{1}^{(m)}=\sum_{i j \alpha}(i+j) M_{i j \alpha}
$$

The concentration of atoms $a$ (calculated for every sublattice separately) is:

$$
\begin{aligned}
& y_{1}^{(1)}=\frac{N_{1}^{(1)}}{N^{(1)}}=\sum_{i j \alpha} \frac{i}{k_{1}} p_{i j \alpha} \\
& y_{1}^{(2)}=\frac{N_{1}^{(2)}}{N^{(2)}}=\sum_{i j \alpha} \frac{j}{k_{2}} p_{i j \alpha}
\end{aligned}
$$

where

$$
\begin{aligned}
p_{i j \alpha} & =M_{i j \alpha} / M \\
1 & =\sum_{i j \alpha} p_{i j \alpha}
\end{aligned}
$$

The just-introduced local per-sublattice concentrations are directly related to the total alloy composition:

$$
x_{1}=\frac{N_{1}}{N}=\sum_{m=1}^{2} \frac{k_{m}}{k} y_{1}^{(m)}
$$


Combinatorial factor $K$ of the system is still given by (8) where this time

$$
\begin{aligned}
\ln K_{1}^{\infty} & =\ln K_{0}=\ln \left(\prod_{m=1}^{2} \frac{N^{(m)} !}{N_{1}^{(m)} ! N_{2}^{(m)} !}\right) \\
& =-\sum_{m=1}^{2} N^{(m)}\left(y_{1}^{(m)} \ln y_{1}^{(m)}+y_{2}^{(m)} \ln y_{2}^{(m)}\right)
\end{aligned}
$$

and

$$
\ln K_{1}=\ln \frac{M !}{\prod_{i j \alpha}\left(M_{i j \alpha}\right) !}=-M \sum_{i j \alpha} p_{i j \alpha} \ln p_{i j \alpha}
$$

The structural parameter $\gamma$, considered in the quasichemical approximation (9), turns out to be equal to 4 .

The energy of the entire system is

$$
\beta \sum_{i j \alpha} U_{i j \alpha} M_{i j \alpha}
$$

and differs from the expression (1) only by using two indexes required to specify the cell composition.

The configurational entropy of the system reaches its maximum when both sublattices have the same composition. The order-disorder transition occurs when the interatomic interaction is strong enough to spontaneously destroy the equality of atomic concentrations.

Accordingly, the variables $y_{1}^{(m)}$ receive dynamically defined values (that can still be uniquely expressed by the formula (48)).

The free energy of the system can be written now as a natural general- 
ization of 10$)$ :

$$
\begin{aligned}
& F\left(N_{1}, N_{2}, \theta ; p_{i j \alpha}, y_{1}^{(m)}, \lambda, \lambda_{0}, \lambda_{m}\right)=\beta M \sum_{i j \alpha} U_{i j \alpha} p_{i j \alpha} \\
& +\theta(1-\gamma) \sum_{m=1}^{2} N^{(m)}\left(y_{1}^{(m)} \ln y_{1}^{(m)}+y_{2}^{(m)} \ln y_{2}^{(m)}\right) \\
& +\theta \gamma M \sum_{i j \alpha} p_{i j \alpha} \ln p_{i j \alpha} \\
& +\lambda_{1} N\left(y_{1}^{(1)}-\sum_{i j \alpha} \frac{i}{k_{1}} p_{i j \alpha}\right)+\lambda_{2} N\left(y_{1}^{(2)}-\sum_{i j \alpha} \frac{j}{k_{2}} p_{i j \alpha}\right) \\
& +\lambda N\left(1-\sum_{i j \alpha} p_{i j \alpha}\right)+\lambda_{0} N\left(x_{1}-\sum_{m=1}^{2} \frac{k_{m}}{k} y_{1}^{(m)}\right)
\end{aligned}
$$

The basic equations of theory can be obtained by minimization of $F$ with respect to $p_{i j \alpha}, y_{1}^{(m)}$ :

$$
\begin{gathered}
\frac{\partial F}{\partial p_{i j \alpha}}=\beta M U_{i j \alpha}+M \theta \gamma \ln p_{i j \alpha}-N \frac{i}{k_{1}} \lambda_{1}-N \frac{j}{k_{2}} \lambda_{2}-N \lambda=0 \\
\frac{\partial F}{\partial y_{1}^{(m)}}=\theta(1-\gamma) N^{(m)}\left(\ln y_{1}^{(m)}-\ln y_{2}^{(m)}\right)+\lambda_{m} N-\lambda_{0} N \frac{k_{m}}{k}=0
\end{gathered}
$$

The first of these equations can be immediately solved in a usual form:

$$
p_{i j \alpha}=\exp \left(-\frac{\beta U_{i j \alpha}}{\theta \gamma}\right) b_{1}^{i} b_{2}^{j} b
$$

where $b$ and $b_{m}$ are defined through

$$
\begin{aligned}
b_{m} & =\exp \left(\frac{\lambda_{m}}{\theta \gamma} \frac{k}{k_{m}}\right) \\
b & =\exp \left(\frac{\lambda k}{\theta \gamma}\right)
\end{aligned}
$$


As always, we introduce now a few additional variables that help to present the equilibrium equations in a traceable form:

$$
p_{i j}=\sum_{\alpha} p_{i j \alpha}=W_{i j} b_{1}^{i} b_{2}^{j} b
$$

where

$$
W_{i j}=\sum_{\alpha} \exp \left(-\frac{\beta U_{i j \alpha}}{\theta \gamma}\right)
$$

so the equations (48) - 49) can be rewritten as:

$$
\begin{aligned}
& y_{1}^{(1)}=\sum_{i j} \frac{i}{k_{1}} W_{i j} b_{1}^{i} b_{2}^{j} b \\
& y_{1}^{(2)}=\sum_{i j} \frac{j}{k_{2}} W_{i j} b_{1}^{i} b_{2}^{j} b
\end{aligned}
$$

where

$$
b=\left(\sum_{i j} W_{i j} b_{1}^{i} b_{2}^{j}\right)^{-1}
$$

To simplify the derived equations we substitute the expressions for $y_{1}^{(m)}$ from (60) into the equation (50):

$$
x_{1}=\sum_{i, j} \frac{i+j}{k} W_{i j} b_{1}^{i} b_{2}^{j} b
$$

This equation has to be combined with the normalization condition 61 to get rid of $b$.

The expression for $x_{1}$ will look simpler if we define a few more combinations:

$$
\begin{aligned}
S_{j} & =\sum_{i} W_{i j} b_{1}^{i} \\
S_{j}^{a} & =\sum_{i} i W_{i j} b_{1}^{i}
\end{aligned}
$$




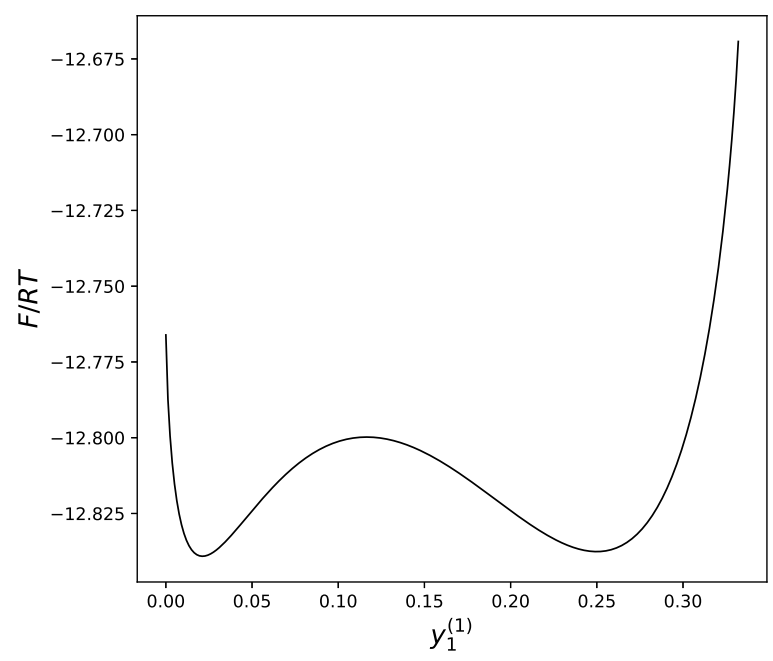

Figure 4: Molar free energy of alloy near the critical point as a function of the concentration of atoms $a$ in the first sublattice: $x_{1}=0.25$ and $\gamma=4.88$.

Finally

$$
x_{1}=\frac{1}{4} \frac{S_{0}^{a}+\left(S_{1}+S_{1}^{a}\right) b_{2}}{S_{0}+S_{1} b_{2}}
$$

Expressing $b_{2}$ from this equation we will have a solution where $b_{1}$ defines distribution of components between sublattices, while $b_{2}$ ensures the correct total composition of the system. The equation (55) (with all components expressed as functions of $b_{1}$ ) defines the values of $y_{1}^{(m)}$ that bring the extreme values to the free energy.

We could numerically solve this remaining equation, but it is more instructive to look at the plot of the free energy of the system versus the concentration of atoms $a$ in the first sublattice. Drawing this plot is an easy task taking into account the described above dependency of both values on $b_{1}$ as a parameter (Fig. 4). 
We can see that at sufficiently low temperatures, the dependency of the free energy on the distribution of atoms between the sub-lattices has two local minima: one, that corresponds to a uniform distribution of atoms over all sub-lattices (near $y_{1}^{(1)}=0.25$ ), and the other, which demonstrates a significant difference in the concentration of atoms between the two sublattices.

The temperature is the main factor that defines which of these two minimums will win the competition, bringing, therefore, the lowest value to the free energy.

Interactive presentation of results of this section is available on GitHub using a Jupyter notebook (https://github.com/EdKr2/TISR6).

\subsection{Cluster Site Approximation}

The results just presented match precisely the results of the theory proposed many years ago Yang (1945); Yang and Li (1947); Li (1949) as generalization of quasichemical theory, and later renamed (unfortunately) as Cluster Site Approximation (CSA) Oates and Wenzl (1996).

It is important to realize that even if the final formulas provided by two theories are identical, the underlying logic and interpretation are quite different.

The method used by TISR is based exclusively on the statistical-thermodynamic approach. Structural elements (cells) introduced by TISR to describe the SRO Kremer (2018a) are used to express the energy and the combinatorics of the system - the two basic units necessary to construct an expression for the free energy. Solutions of equations (54) and (55), derived by the free energy minimization, can be presented either in parametric or in numeric 
form and constitute the output of the theory.

In particular, the equation (56) is part of this output.

CSA uses another approach. It is based on an ansatz that is enforced into the theory under the artificial assumption of similarity of clusters behavior with the behavior of gaseous molecules Yang (1945); Yang and Li (1947); Li (1949). The equation (56), which was directly derived using TISR, is only postulated inside CSA approach.

It comes finally to the same results as TISR but simultaneously turns the theory into a very complex and difficult to trace method.

The equivalence of the two theories was shown in detail in Kremer (2020c) and will be shortly reviewed here.

Since the expression (56) for $p_{i j \alpha}$ is postulated by CSA, this variable has to be excluded from the free energy functional (53), if we should like to follow the CSA logic.

It means, that technically the transfer from the equations of TISR to equations of CSA can be achieved by direct substitution of (56) into (53).

Even better, we can achieve the same result by multiplying the middle part of the equation (54) by $p_{i j \alpha}$ and extracting the result (that is still equal 
to zero) from the free energy expression (53):

$$
\begin{aligned}
& F\left(N_{1}, N_{2}, \theta ; b_{m}\right)= \\
& \theta(1-\gamma) \sum_{m=1}^{2} N^{(m)}\left(y_{1}^{(m)} \ln y_{1}^{(m)}+y_{2}^{(m)} \ln y_{2}^{(m)}\right) \\
& +\sum_{m=1}^{2} \lambda_{m} N y_{1}^{(m)}+\lambda N \\
& +\lambda_{0} N\left(x_{1}-\sum_{m=1}^{2} \frac{k_{m}}{k} y_{1}^{(m)}\right)
\end{aligned}
$$

where all dynamic variables on the right site are assumed now to be expressed through $b_{m}$ using (57) and (60).

As mentioned, the free energy expression (65) is identical to the corresponding expression from Oates et al. (1999) (equation (5)). It becomes evident if we use the presented in the table 2 correspondence list between

\begin{tabular}{|c|c|c|}
\hline This text & Oates et al. (1999) & Meaning \\
\hline $\mathrm{k}$ & $\mathrm{n}$ & count of atoms in a cell \\
\hline $\mathrm{m}$ & $\mathrm{p}$ & count of interatomic links in a cell \\
\hline$y_{1}^{(m)}$ & $y_{A}^{m}$ & concentration of atoms $a$ in sublattice $m$ \\
\hline$k_{m} / k$ & $f_{m}$ & sublattice fraction \\
\hline$\gamma=z k / 2 m$ & $z n / 2 p$ & structural parameter of theory \\
\hline $\ln b_{m}=\lambda_{m} k / \theta \gamma k_{m}$ & $\mu_{A}^{m}$ & Lagrangian multiplier \\
\hline
\end{tabular}
the notations used in the current article and the notations introduced by Oates et al. (1999).

Table 2: Notation correspondence between Oates et al. (1999) and the current article. 
Significant improvements to CSA were introduced by Oates et al. (1999) as an attempt to resolve difficulties with the applications of theory. In order to reproduce the results of Monte-Carlo experiments Ferreira et al. (1998) Oates Oates et al. (1999) decided to use some of the internal parameters of theory as adjustable.

Surprisingly, this purely formal experiment should be considered very successful.

As shown in Kremer (2019) and briefly reviewed in section 4, $\gamma$ is a element of TISR, which can be selected to reproduce the correct critical temperature of transformation and significantly improve the accuracy of calculations.

This parameter exists also inside CSA (maybe, under another name).

Oates modified this parameter to bring in the consistence the results of CSA calculations and Monte-Carlo experiments Ferreira et al. (1998).

The resulting value was estimated as $\gamma \approx 4.88$ (rather than quasichemical value 4.0). This value was used to create Fig. 4.

As clear from the above, this number is the only justified value for $\gamma$ if we are interested to consider the thermodynamics of the FCC lattice close to the critical temperatures. Unfortunately, it was decided to continue to treat $\gamma$ in the future just as a dummy adjustable parameter.

As a result of this approach, the structural parameter $\gamma$ had been varied in Oates and Wenzl (1996); Oates et al. (1999); Oates (2007); Zhu et al. $(2010)$ in the region from 4 to 7 totally neglecting the real meaning of this quantity.

Using $\gamma$ as a dummy adjustable parameter may help to bring theoretical 
curve closer to the experimental data but the physical meaning of theory will be dimmed, while the physically significant quantities will be shifted away from the correct values.

It looks that the importance of correct value for $\gamma$ was never clearly formulated.

A more detailed juxtaposition of two theories can be found in Kremer (2020c) together with the critical analysis of some popular statements that may still cause questions.

\section{Multicomponent Systems}

The multicomponent generalization of thermodynamic models is an important task that can cause significant mathematical difficulties, and these difficulties usually increase with the number of components.

For example, we discussed in section 3 the explicit solution $(22)$ found for the quasichemical model in a two-component system. Generalization of this model even for ternary alloys produces a system of nonlinear equations that allow only a numerical solution Mickeleit and Lacmann (1983).

The same problem exists in the case of multicomponent ASM Pok-Kai et al. (1982) where even the binary system formalism cannot avoid usage of nonlinear equations.

The multicomponent generalization of ASM encounters even more principal difficulties when attempting to define multicomponent associates and the interaction between them Sundman et al. (2018).

In contrast, the parametric representation of a solution using TISR makes multicomponent generalization almost trivial in many cases. Usually it re- 
quires only the replacement of the index $i$, that describes the cell composition in the binary system, by a $n$-component vector:

$$
\mathbf{i}=\left(i_{1}, i_{2}, \ldots, i_{n}\right)
$$

where

$$
i_{1}+i_{2}+\cdots+i_{n}=k
$$

As result, we have the following expression for the energy:

$$
E=\beta \sum_{\mathbf{i} \alpha} U_{\mathbf{i} \alpha} M_{\mathbf{i} \alpha}
$$

where

$$
\alpha=1,2, \ldots\left(\begin{array}{l}
k \\
\mathbf{i}
\end{array}\right)=1,2, \ldots\left(\begin{array}{c}
k \\
i_{1}, i_{2}, \ldots, i_{n}
\end{array}\right)
$$

The composition of the alloy is then

$$
N_{c}=\sum_{\mathbf{i} \alpha} i_{c} M_{\mathbf{i} \alpha}, \quad c=1,2, \ldots n
$$

The combinatorial factor is still expressed by (8) where this time

$$
K_{0}=\frac{N !}{\prod_{c=1}^{n} N_{c} !}
$$

Free energy can be written as:

$$
\begin{aligned}
F & =\beta \sum_{\mathbf{i} \alpha} U_{\mathbf{i} \alpha} M_{\mathbf{i} \alpha}-\theta(1-\gamma) \ln K_{0}-\theta \gamma \ln K_{1} \\
& +\sum_{c} \lambda_{c}\left(N_{c}-\sum_{\mathbf{i} \alpha} i_{c} M_{\mathbf{i} \alpha}\right)
\end{aligned}
$$

Omitting the standard steps, we receive: 


$$
p_{\mathbf{i}}=\sum_{\alpha} p_{\mathbf{i} \alpha}=W_{\mathbf{i}} b_{1}^{i_{1}} b_{2}^{i_{2}} \cdots b_{n}^{i_{n}}
$$

while the expression for the chemical potential (14) remains unchanged.

Thanks to the condition (66), all the physically meaningful values depend effectively on $n-1$ independent variables, ensuring the parametric presentation of properties.

This short review makes the details of multicomponent generalization totally clear in the case when all knots inside a cell are equivalent.

If a cell has to be divided into two (or more) sub-cells, we need more than one vector index to describe such cell. The key equation (58) will be presented now as

$$
p_{\mathbf{i j}}=\sum_{\alpha} p_{\mathbf{i j} \alpha}=W_{\mathbf{i j}} b b_{1}^{i_{1}} d_{1}^{j_{1}} b_{2}^{i_{2}} d_{2}^{j_{2}} \cdots
$$

The presence of two vector variables leads to more cumbersome equations, however, the main logic remains unchanged.

\section{Conclusions}

Unification of several model theories inside some general scheme presents a challenging and important problem.

This article tries to achieve this target by presenting the combinatorial factor of theory in a new form, that is more intuitive, more general, and allows a more strong precision control than the traditionally used constructs.

The developed approach allows to use the same formalism to reproduce the results of multiple theories: quasichemical theory, associated solution model, surrounded atom model, cluster site approximation. 
It has been shown that just by choosing a specific for each model group of atoms - cells - we can reproduce - or even significantly improve - the results of the listed theories.

Remarkably, that in all cases the expressions for the energy and for the combinatorial factor remain basically the same. What is changing, is just the set of indexes necessary to identify the composition and the structure of every cell, and a set of external restrictions that have to be added to the free energy functional to fix the total composition and (in some cases) ensure the internal consistency of the formalism.

An unusual feature of the theory is the presentation of results in a parametric form, where both thermodynamic values and component concentrations are expressed as functions of a common parameter (or several parameters, in the case of a multicomponent system) that naturally arise as part of the solution.

It occurs, surprisingly, that just usage of this parametric form dramatically simplifies formalism, especially the multicomponent generalizations of theory.

Developed as part of theory combinatorial factor proved its advantages by helping to find and correct some of the errors that are found within the associated solution model and within the surrounded atom model. The identified errors, if not being fixed, could compromise the accuracy and physical interpretation of these theories.

Moreover, the new combinatorial factor, which without any tweaks provides the accuracy of calculations at the level of quasichemical theory, allows also some modifications that ensure the reproduction of the correct critical 
temperature of alloy and significantly improves the accuracy of all thermodynamic values.

Finally, very important for the potential application of TISR is the dramatic reduction in the number of adjustable parameters required for the description of thermodynamic properties of alloys - the same feature, that was first demonstrated by cluster site approximation.

For example, when considering FCC lattice in tetrahedron approximation we need:

- Three temperature-dependent parameters to describe a binary system;

- Three additional temperature-dependent parameters to describe the comprising ternary system;

- One additional temperature-dependent parameter to describe the comprising quaternary system;

- No additional parameters for the systems with five or more components.

We can expect that the possibility to apply this economic approach to a wide range of systems creates a large space for potential applications of theory.

\section{Acknowledgment}

This research did not receive any specific grant from funding agencies in the public, commercial, or not-for-profit sectors.

\section{References}

Paolo Ossi. Disordered materials: an introduction. Springer Science \& Business Media, 2010. 
Alexandr I. Gusev, Andrej A. Rempel, and Andreas J. Magerl. Disorder and Order in Strongly Nonstoichiometric Compounds. Springer Berlin Heidelberg, 2001.

Bo Sundman, Qing Chen, and Yong Du. A review of calphad modeling of ordered phases. Journal of Phase Equilibria and Diffusion, 39(5):678-693, aug 2018. URL https://doi.org/10.1007/s11669-018-0671-y.

M.A. Krivoglaz and A.A. Smirnov. The Theory of Order-disorder in Alloys [Translated from the Original Russian by Scripta Technica. Edited by Bruce Chalmers.]. Macdonald, 1964.

Srikumar Banerjee and Pradip Mukhopadhyay. Phase transformations: examples from titanium and zirconium alloys. Elsevier, 2010.

José L Morán-López and José M Sánchez, editors. Theory and applications of the cluster variation and path probability methods, 2012. Springer Science \& Business Media. ISBN 9781461304197.

Louis A. Girifalco. Statistical Mechanics of Solids. Oxford University Press, 2000. ISBN 9780195119657.

Michel Soustelle. Modeling of Liquid Phases (Chemical Thermodynamics Set). Wiley-ISTE, aug 2015. ISBN 1848218656.

C. H. P. Lupis. Chemical Thermodynamics of Materials. Prentice Hall, 1983. ISBN 9780444007131.

WA Oates and H Wenzl. The cluster/site approximation for multicomponent solutions - a practical alternative to the cluster variation method. Scripta 
materialia, 35(5), 1996. URL https://doi .org/10.1016/1359-6462(96) 00198-4.

E. Kremer. Theory of Inhomogeneous Short Range Order and its Usage for Description the Thermodynamic Properties of Sulfide Melts of Iron, Cobalt, and Nickel (In Russian). PhD thesis, Leningrad State University, Russia, 1988.

Edward Kremer. Theory of Inhomogeneous Short Range Order and Calphad Modeling. Part 1. Basic Formalism. Jul 2018a. URL https://doi.org/ 10.31219/osf.io/cs47d.

C. Domb. Ising model. In Phase Transitions and Critical Phenomena, Volume 3, pages 357-485. Academic Press, 1974. ISBN 0122203038.

Edward Kremer. Theory of Inhomogeneous Short Range Order and Calphad Modeling. Part 4. Improved Combinatorial Factor - osf.io preprint. Nov 2019. URL https://doi.org/10.31219/osf .io/zmwcv.

E.A. Guggenheim. Mixtures. Oxford University Press, 1952.

Edward Kremer. Theory of Inhomogeneous Short Range Order and Calphad Modeling. Part 2. Quasichemical Theory - osf.io preprint. Sep 2018b. URL https://doi.org/10.31219/osf .io/4hzf9.

T. L. Hill. Statistical Mechanics. McGraw-Hill Book Company, Inc., 1956.

Edward Kremer. Theory of Inhomogeneous Short Range Order and Calphad Modeling. Part 3. Association Solution Model Reconstructed - osf.io preprint. Nov 2018c. URL https://doi.org/10.31219/osf .io/7x2e4. 
Lars Onsager. Crystal Statistics. I. A Two-Dimensional Model with an OrderDisorder Transition. Physical Review, 65(3):117-149, Jan 1944. URL https://doi.org/10.1103/physrev.65.117.

I. Prigogine, L. Mathot-Sarolea, and L. Van Hove. On the combinatory factor in regular assemblies. Transactions of the Faraday Society, 48:485, 1952. URL https://doi .org/10.1039/tf9524800485.

Hans Lukas. Computational Thermodynamics: The Calphad Method. Cambridge University Press, aug 2007. ISBN 0521868114.

Jean-François Sadoc. Geometrical Frustration. Cambridge University Press, oct 1999. ISBN 0521441986.

Jean-Claude Mathieu, Françis Durand, and Étienne Bonnier. L'atome entouré, entité de base d'un modèle quasichimique de solution binaire. Journal de Chimie Physique, 62:1289-1296, 1965. URL https://doi.org/10. $1051 / \mathrm{jcp} / 1965621289$.

P. Hicter, J.C. Mathieu, F. Durand, and E. Bonnier. A model for the analysis of enthalpies and entropies of liquid binary alloys. Advances in Physics, 16(63):523-533, July 1967. URL https://doi.org/10.1080/ 00018736700101635 .

J. Lehmann. Application of ArcelorMittal maizières thermodynamic models to liquid steel elaboration. Revue de Métallurgie, 105(11):539-550, November 2008. URL https://doi.org/10.1051/metal:2008070.

J. Lehmann and L. Zhang. The generalized central atom for metallurgical 
slags and high alloyed steel grades. steel research international, 81(10):875879, September 2010. URL https://doi .org/10.1002/srin.201000137.

Do Chieu Ha and Nguyen Nhat Khanh. The surrounded atom theory of orderdisorder phase transition in binary alloys. Communications in Physics, 21 (3):265, September 2011. URL https://doi.org/10.15625/0868-3166/ $21 / 3 / 177$.

Natalia Kotova, Natalia Golovata, and Natalia Usenko. Calculation of thermodynamic properties of liquid Fe-Ln alloys. French-Ukrainian Journal of Chemistry, 3(2):40-43, 2015. URL https://doi.org/10.17721/ fujcv3i2p40-43.

Edward Kremer. Theory of Inhomogeneous Short Range Order and Calphad Modeling. Part 7. Surrounded Atom Model Reconstructed - osf.io preprint. Aug 2020a. URL https://doi.org/10.31219/osf .io/yrx5g.

Edward Kremer. Theory of Inhomogeneous Short Range Order and Calphad Modeling. Part 5. Extended Formalism - osf.io preprint. Jun 2020b. URL https://doi.org/10.31219/osf .io/k86gc.

Madan Lal Kapoor. An approach to thermodynamics of binary substitutional solutions. Transactions of the Japan Institute of Metals, 19(10):519-529, 1978. URL https://doi .org/10.2320/matertrans1960.19.519.

C Bichara, C Bergman, and J.-C Mathieu. Monte carlo calculations of thermodynamic properties of alloys in the case of the surrounded atom model. Acta Metallurgica, 33(1):91-95, January 1985. URL https: //doi.org/10.1016/0001-6160(85)90222-6. 
Arthur D. Pelton and Youn-Bae Kang. Modeling short-range ordering in solutions. International Journal of Materials Research, 98:907-917, 2007. URL https://doi .org/10.3139/146.101554.

C. N. Yang. A generalization of the quasi-chemical method in the statistical theory of superlattices. The Journal of Chemical Physics, 13(2):66-76, February 1945. URL https://doi .org/10.1063/1.1724001.

C. N. Yang and Y. Li. General Theory of the Quasi-Chemical Method in the Statistical Theory of Superlattices. Chinese Journal of Physics, 11(2): 59-71, Oct 1947.

Yin-Yuan Li. Quasi-chemical theory of order for the copper gold alloy system. The Journal of Chemical Physics, 17(5):447-454, 1949. URL https:// doi.org/10.1063/1.1747287.

Edward Kremer. Theory of Inhomogeneous Short Range Order and Calphad Modeling. Part 6. Order-Disorder Transformations in Alloys - osf.io preprint. Jul 2020c. URL https://doi.org/10.31219/osf .io/ja9mn.

W. A. Oates, F. Zhang, S-L. Chen, and Y. A. Chang. Improved cluster-site approximation for the entropy of mixing in multicomponent solid solutions. Physical Review B, 59(17):11221-11225, May 1999. URL https://doi. org/10.1103/PhysRevB.59.11221.

Luiz G. Ferreira, C. Wolverton, and Alex Zunger. Evaluating and improving the cluster variation method entropy functional for ising alloys. The Journal of Chemical Physics, 108(7):2912-2918, February 1998. URL https://doi.org/10.1063/1.475695. 
WA Oates. Configurational entropies of mixing in solid alloys. Journal of phase equilibria and diffusion, 28(1):79-89, 2007. URL https://doi .org/ $10.1007 / \mathrm{s} 11669-006-9008-3$.

J Zhu, C Zhang, D Ballard, P Martin, J Fournelle, W Cao, and YA Chang. Study of the Ni-rich multi-phase equilibria in $\mathrm{Ni}-\mathrm{Al}-\mathrm{Pt}$ alloys using the cluster/site approximation for the face-centered cubic phases. Acta materialia, 58(1):180-188, 2010. URL https://doi.org/10.1016/j.actamat. 2009.08 .068

Michael Mickeleit and Rolf Lacmann. Statistical mechanics and thermodynamic properties of liquid multicomponent mixtures. Part I. The Taylor series for quasichemical equilibrium of ternary mixtures. Fluid Phase Equilibria, 12(3):201-216, January 1983. URL https://doi.org/10.1016/ 0378-3812(83)80062-4.

Liao Pok-Kai, Su Ching-Hua, Tung Tse, and R.F. Brebrick. Quantitative simultaneous fit to the liquidus surface and thermodynamic data for the Ga-In-Sb system using an associated solution model for the liquid. Calphad, 6(2):141-169, April 1982. URL https://doi.org/10.1016/ 0364-5916(82)90009-8. 